\title{
PoSSO - Physics of SubStellar Objects
}

Hugh R.A. Jones ${ }^{1}$, Serena Viti ${ }^{2}$, Jonathan Tennyson ${ }^{2}$, Bob Barber ${ }^{2}$, Juliet C. Pickering $^{3}$, Richard Blackwell-Whitehead ${ }^{3}$, Jean-Paul Champion ${ }^{4}$, France

Allard $^{5}$, Peter H. Hauschildt ${ }^{6}$, Uffe G. Jorgensen ${ }^{7}$, Pascale Ehrenfreund ${ }^{8}$, Ewa Stachowska $^{9}$, Hans-Gunter Ludwig ${ }^{10}$, Yakiv Pavlenko ${ }^{11}$, Yuri Lyubchik ${ }^{11}$, and Robert L. Kurucz ${ }^{12}$

1 Astrophysics Research Institute, Liverpool John Moores University, UK

2 Department of Physics and Astronomy, University College London, UK

3 Blackett Laboratory, Imperial College, London, UK

4 Laboratoire de Physique, Universite of Bourgogne, Dijon, France

5 Centre de Recherche Astronomique de Lyon, Lyon, France

6 Hamburger Sternwarte, Hamburg, Germany

7 Niels Bohr Institute, Copenhagen, Denmark

8 Universitet van Amsterdam, Amsterdam, The Netherlands

9 Poznan University of Technology, Poland

10 Lund Observatory, Lund, Sweden

11 Main Astronomical Observatory, Kyiv, Ukraine

12 Centre for Astrophysics, Harvard Smithsonian Center for Astrophysics, USA

\begin{abstract}
A full understanding of the properties of substellar objects is one of the major challenges facing astrophysics. Since their discovery in 1995, we have discovered hundreds of brown dwarfs and extrasolar planets. While these discoveries have enabled important comparisons with theory, observational progress has been much more rapid than the theoretical understanding of cool atmospheres. The determination of mass, abundances, gravities and temperatures is not yet possible. The key problem is that substellar objects emit their observable radiation in the infrared region of the spectrum where our knowledge of atomic, molecular and line broadening data is poor. In order to understand these objects, and extra-solar planets increasing more like those our Solar System, we urge the wider physical chemistry community to engage in this exciting new field. Here we sketch an outline of the atoms, molecules and processes requiring study.
\end{abstract}

\section{Introduction}

At a Cool Stars meeting in Florence, Italy in 1995 the field of substellar astrophysics was born. The discovery of definitive signals from both the brown dwarf GJ229B (Nakajima et al. 1995) and the extra-solar planet 51 Peg b (Mayor \& Queloz 1995 were announced. The low luminosity and strong methane bands shown for GJ229B indicated a temperature of around $1000 \mathrm{~K}$ and luminosity of $10^{-5}$ that of the Sun. No plausible star can be so cool and faint. The acceptance of brown dwarfs by the community was immediate. While the discovery of the first brown dwarf was extremely exciting coming more than 30 years after the 
prediction of then so-called 'dark stars' by Kumar (1962) and Hayashi (1961), it was somewhat eclipsed by the announcement of the first extra-solar planet. In fact the announcement of the discovery of an extra-solar planet had been made many times (e.g., Bell 2001) in the 400 years since Giordano Bruno was executed in 1584 for promoting the idea that there might be other worlds around other stars. The 1995 announcement by Mayor \& Queloz (1995) was different; it was almost immediately confirmed and added to by Marcy \& Butler (1996). The full acceptance of extra-solar planets has taken a little longer with confirmation of $51 \mathrm{Peg} b$ by many groups and the discovery of extra-solar planets being made by several different techniques: HD209458 by radial velocity and transit (Henry et al. 2000; Charbonneau et al. 2000); GJ876bc by radial velocity and astrometry (Benedict et al. 2002).

In addition to bona fide brown dwarfs and extrasolar planets the discovery has probably also been made of another class of objects straddling brown dwarfs and planets - so called planetary mass objects or sub-brown dwarfs (e.g., Tamura et al. 1998; Oasa et al. 2003). Thus we can now study more than 100 extra-solar planets, 300 brown dwarfs and some 100 sub brown dwarfs. Unfortunately the interpretation of their infrared spectra, generally the only spectral region where they are bright enough to observe, is badly hampered by a lack of high quality input data in the infrared for the modelling of their atmospheres. Apart from these relatively newly discovered objects the lack of infrared data is also an issue

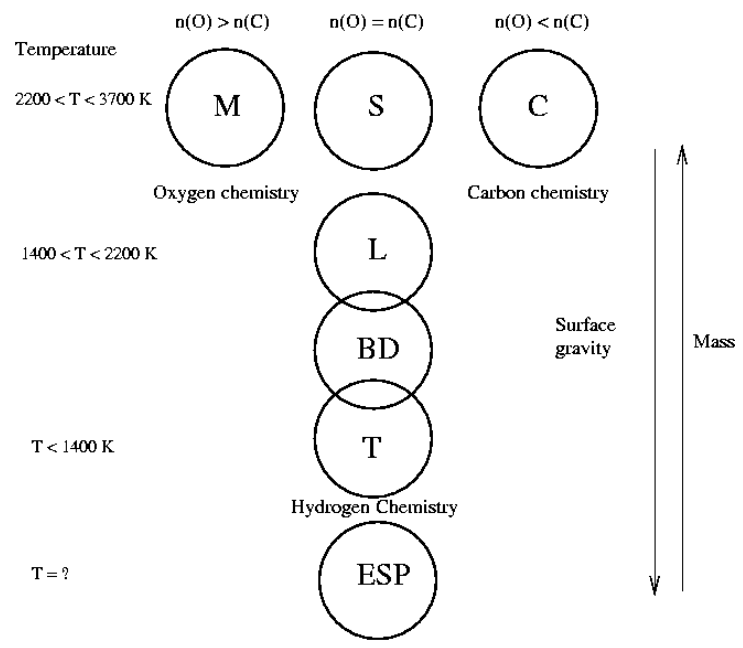

Fig. 1. The molecular composition of stellar atmospheres divides into two classes: oxygen-rich and the less common carbon-rich. There is also a third rare class of S stars where carbon and oxygen abundances are close to unity. In the objects of low-mass objects most of the hydrogen is locked in $\mathrm{H}_{2}$ and most of the carbon in $\mathrm{CO}$ for LMS and $\mathrm{BD}$, and $\mathrm{CH}_{4}$ for $\mathrm{BD}$ and ESP, with excess oxygen for oxygen-rich objects bound in molecules such as $\mathrm{TiO}, \mathrm{VO}$ and $\mathrm{H}_{2} \mathrm{O}$, and with excess carbon for carbon-rich objects, bound in molecules such as $\mathrm{HCN}, \mathrm{CH}_{4}$ and $\mathrm{HCCH}$. 
for the study of M giants (the dominant population by luminosity of elliptical galaxies), $\mathrm{S}$ stars (where the carbon and oxygen abundances are similar) and $\mathrm{C}$ stars (where the carbon abundance dominates over Oxygen). A part from wanting to understand the properties of our cool neighbours (e.g. Fig. 1), there are general questions relating to cool stars from cosmology (are some of the low mass stars primordial?), from star formation (does the initial mass function vary among star formation regions, and is there a lower mass limit below which no objects form?) and Galactic dynamics (how much mass is stored in substellar objects?).

\section{Low-mass stars, brown dwarfs and extrasolar planets}

There are significant unresolved issues in the field of low-mass stars (LMS), brown dwarfs (BD) and extra-solar planets (ESP) that hinder our understanding of these objects. Examples include: (1) The effective temperature scale and its correlation with spectral type are not yet properly determined: some objects which fall within the same spectral type show substantial differences in their energy distribution and this suggests different effective temperatures; (2) metallicity and surface gravity (and hence ages) remain controversial: colour-colour diagrams, usually used as indicators, do not yet reproduce the broad-band fluxes within a reasonable error. An alternative and complementary way of determining both metallicity and surface gravity is spectroscopically by comparing observed

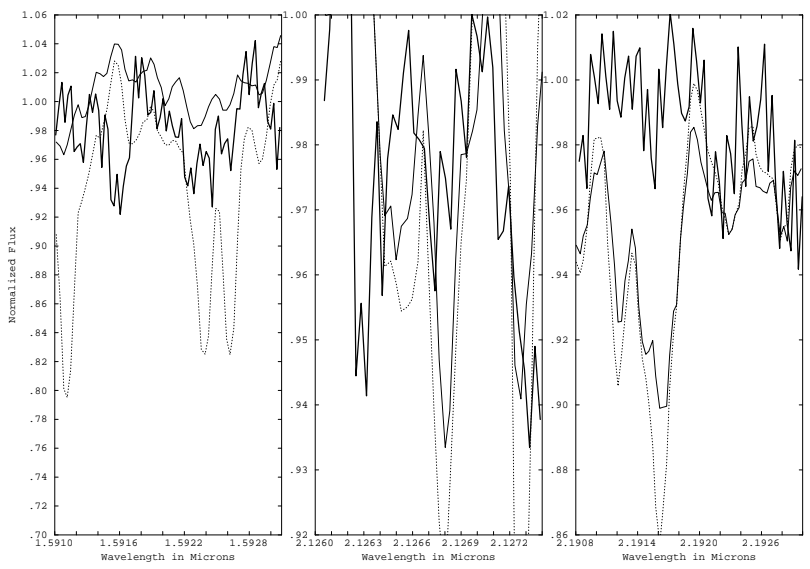

Fig. 2. Spectra for the lowest mass eclipsing binary star CM Draconis compared with synthetic spectra for features selected to be of high sensitivity to model parameters (from Viti et al. 2002): thick continuous line - CM Dra; thin continuous line - 3100 $\mathrm{K},[\mathrm{M} / \mathrm{H}]=-1.0$ model; dotted line $-3100 \mathrm{~K},[\mathrm{M} / \mathrm{H}]=0.0$ model. The generally poor fit of atomic lines between synthetic and observed spectra is typical for cool dwarfs. The strong atomic features on the left, centre and right panels are respectively Si I $(\lambda \sim$ $1.5931 \mu \mathrm{m}$, appearing at $\sim 1.5925 \mu \mathrm{m})$, Al I $(\lambda \sim 2.1272 \mu \mathrm{m}$, appearing at $\sim 2.1264$ $\mu \mathrm{m})$ and Ti I $(\lambda \sim 2.1925 \mu \mathrm{m}$, appearing at $\sim 2.1916 \mu \mathrm{m})$. 
with synthetic spectra. At present the poor quality of the data available for this leads to fits where the temperature and metallicity are too correlated to be reliably determined independently (e.g. Fig. 2) . (3) brown dwarfs do not burn hydrogen but, depending on mass, may burn D or Li. These burning limits, as potentially determined by the 'lithium test' and the 'deuterium test', could, given the necessary spectroscopic data, be used to determine the true substellar nature of ultracool dwarfs; (4) brown dwarf formation: how can a brown dwarf form when its mass is at least 10 times smaller than the typical Jeans mass in star forming molecular clouds? Once it has formed, how can it avoid substantial further accretion of gas? What prevents them exceeding the hydrogen burning mass limit? (5) The distinction between ESPs and BDs; some BDs are less massive than the most massive planetary companions (known as sub brown dwarfs or planetary mass objects); hence the use of a simple mass-based definition to distinguish between stars and planets is dubious. (6) The trajectory of metalfree stars on the HR diagram: recent observations of highly metal deficient dwarf stars raise the question whether such stars are really primordial ones which have accreted some metals or are the results of the first supernovae explosions. (7) At present most known ESPs are larger than Jupiter but orbit close to their star so they are strongly irradiated. Assuming these planets are gas giants, what is their chemical structure and thermal composition? Even more importantly, do they exhibit a clear spectral signature? (8) Models of variable stars, e.g. Mira:

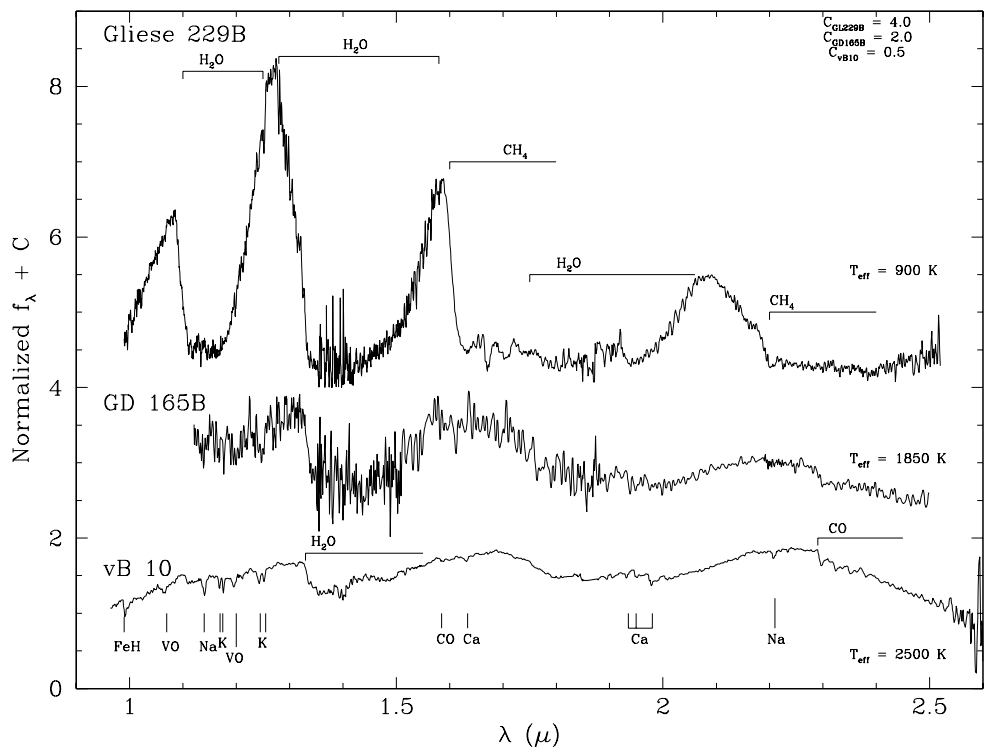

Fig. 3. The transition from $M$ to $T$ dwarf spectra across the infrared region showing the primary molecular and atomic absorption features (from Oppenheimer et al. 1999). 
present models underestimate the mass loss rates and these models are particularly sensitive to the input molecular data.

Our understanding of all these problems, and others, depends heavily on the improvement of atmospheric and evolutionary models. Advances in atmospheric modelling of LMS, BDs and ESPs have been slowed down by three main unresolved issues: (1) inaccurate and incomplete treatment for opacity; (2) the lack of an accurate equation of state; (3) inaccurate (by more than $50 \%$ at times) oscillator strengths of important atomic spectral markers.

Table 1. Atoms, molecules, dust compounds and mechanisms relevant for cool objects. We have made our selection of atomic lines based on Lyubchik et al. (2004). The bold species show immediate priorities for PoSSO. The species in italic denote those for which we have measurements in hand from our PPARC and Royal Society programmes (e.g., Blackwell-Whitehead et al. 2004).

\begin{tabular}{|c|c|c|}
\hline Metal-free & Oxygen-rich & Carbon-rich \\
\hline $\begin{array}{l}\mathrm{HeH}^{+}, \mathrm{H}_{3}^{+}, \mathbf{L i H}, \mathrm{H}_{2}, \\
\mathrm{H}^{-}, \text {collision-induced } \\
\text { absorption by } \mathrm{H}_{2}, \\
\text { Molecular line } \\
\text { broadening }\end{array}$ & $\begin{array}{l}\mathrm{H}_{2}, \mathrm{H}_{3}^{+}, \mathrm{H}_{2} \mathrm{O}, \mathrm{TiO}, \\
\mathrm{CO}, \mathrm{CH}, \mathrm{OH}, \mathrm{YO}, \\
\mathrm{VO}, \mathrm{MgH}, \mathrm{FeH}, \\
\mathrm{CaH}, \mathrm{AlH}, \mathrm{SiH}, \mathrm{TiH}, \\
\mathrm{AlO}, \mathrm{MgO}, \mathrm{dust} \\
\left(\mathrm{Al}_{2} \mathrm{O}_{3}, \mathrm{CaTiO}_{3}, \mathrm{Fe},\right. \\
\mathrm{MgSiO}_{3}, \mathrm{Mg}_{2} \mathrm{SiO}_{4}, \\
\left.\mathrm{ZrO}_{2}\right), \mathrm{Na}, \mathrm{Fe} \mathrm{I}, \mathrm{Ni} \text { I, } \\
\mathrm{Ti} I, \mathrm{Mg} \mathbf{I}, \mathrm{Al} \mathrm{I}, \mathrm{Cr} \mathbf{I}, \\
M n I, \text { Molecular line } \\
\text { broadening }\end{array}$ & $\begin{array}{l}\mathrm{H}_{2}, \mathrm{CO}, \mathrm{HCN}, \mathrm{CN}, \\
\mathrm{CH}, \mathrm{CS}, \mathbf{C H}_{4}, \mathbf{C}_{3}, \\
\mathbf{C}_{2} \mathbf{H}_{2}, \mathbf{A l H}, \text { dust, } \\
\text { Molecular line } \\
\text { broadening }\end{array}$ \\
\hline
\end{tabular}

\section{Requirements for opacities, equation of state and line characteristics}

The main sources of opacity for LMS, BD and ESP are open shell diatomics (such as $\mathrm{TiO}$ ), polyatomic molecules (such as $\mathrm{H}_{2} \mathrm{O}$ ) and dust. Although most of the molecules that play an important role in the opacity of these objects have been known since the early 1930s, in the absence of detailed lists of transitions for them, it has been necessary to resort to average opacities such as the Just Overlapping Line Approximation, Straight Mean or Harmonic mean techniques, which approximate (by a continuum distribution) the absorption within a band 
or a predefined wavelength bin. While computationally economical, they make the assumption that the rotational fine structure is smeared out, ie., the lines overlap without being saturated. Such conditions are never truly met even for the strongest bands of $\mathrm{TiO}$ and $\mathrm{H}_{2} \mathrm{O}$ in the densest of the LMS atmospheres and these models tend to overestimate the resulting molecular blanketing by trapping photons that would otherwise have escaped from between the lines. A far more accurate account of molecular and atomic opacities in model atmospheres is achieved by applying an Opacity Sampling (OS) treatment of transition lists on a prescribed fine grid of wavelengths. However, even with great improvements in the treatment of opacities for some species (e.g., $\mathrm{CO}$, TiO), we still lack accurate opacities for important absorbers such as $\mathrm{H}_{2} \mathrm{O}$ and $\mathrm{CH}_{4}$ (e.g., Fig. 3). Another strong source of opacity is the condensations of molecules and grains: the impact of condensation on the spectral distribution and atmospheres of LMS, BD and ESP is to gradually deplete the gas phase of titanium, iron, vanadium and oxygen. Models need to include both grain formation and grain opacities: present models have shown that the inclusion of opacities of corundum, enstatite and iron heat the photospheric layers and change the overall structure of the atmosphere. Yet the state of art dusty models are still coarse and better grain opacities which include equilibrium condensation abundances, effects of distribution of grain sizes and shape are needed.

The equation of state (EOS), i.e., the relations between the density and temperature of the material on the one hand and its pressure and internal energy, specific heats, etc, on the other hand is for most stars quite simple as most of the gas consists of hydrogen and other light elements, and these have lost most of their electrons. However, in the case of LMS, non-ideal effects, such as Coulomb interactions become important. It is then necessary to use a more adequate equation where all the relevant atoms and molecules are included and partition functions for all these species need to be accurately known. The development of a detailed EOS is necessary to calculate the occupation numbers needed for opacity calculations; for example, for ultracool dwarfs it is essential to have accurate abundances of species such as deuterium since the deuterium burning limit may be where the separation between BDs and ESP lie. Hence, as most of the deuterium would be locked in HDO, an accurate partition function for this species is desirable.

While atomic features make little difference to the opacity of these objects, they are extremely important spectral markers and potentially excellent diagnostics for effective temperature, metallicity and surface gravity. A further issue is line broadening. The strongest lines can be very broad owing to van der Waals pressure broadening due to the interaction between two different neutral particles. No exact method has yet been developed for the case of collisions of atoms and molecules with the molecular hydrogen that dominates the atmospheres of BD and ESP. The production of reliable synthetic spectra thus also requires the use of complete linelists as well as a systematic theory of line broadening. 


\section{Acknowledgement}

We are very grateful to PPARC and the Royal Society who are funding our programme of atomic measurements and travel to and from Kyiv. We have applied to the Sixth Framework programme of the European Union for a Marie Curie Research Training Network to broaden the work of the project.

\section{References}

1. Bell G.H., 2001, www.public.asu.edu/s̃ciref/exoplnt.htm

2. Blackwell-Whitehead et al., 2004, A\&A, submitted

3. Benedict et al., 2002, ApJ, 581, L115

4. Butler R.P., Marcy G.W., 1996, ApJ, 464, L153

5. Charbonneau et al. 2000, ApJ, 529, L45

6. Hayashi C., 1961, PASJ, 13, 450

7. Henry et al., 2000, ApJ, 529, L41

8. Kumar S.S., 1962, AJ, 67, 579

9. Lyubchik et al. 2004, A\&A, 416, 655

10. Mayor M., Queloz D., 1995, Nature, 378, 355

11. Nakajima et al., 1995, Nature, 378, 463

12. Oasa Y., 2003, IAU Symposium 211, p.91, ed. Martin E.

13. Oppenheimer B., 1999, PhD, California Institute of Technology

14. Tamura et al. 1998, Science, 282, 1095

15. Viti et al., 2002, MNRAS, 329, 290 